\title{
WILD UNIVERSALLY PIERCED ARCS
}

\author{
HARVEY ROSEN
}

\begin{abstract}
We show that each arc in $E^{3}$ with two shrinking points is universally pierced. Examples of universally pierced arcs and a universally pierced simple closed curve are given.
\end{abstract}

1. Introduction. In [13], an example of a wild set $A$ in $E^{3}$ is given with the property that each 2 -sphere which contains $A$ and is locally tame modulo $A$ can be pierced by a tame arc at each point of $A$. In this paper, the following special examples with this same property are given: (1) $X_{1}$ is a wild arc with two shrinking endpoints and $E^{3}-X_{1}$ is 1-ALG at one endpoint of $X_{1}$; (2) $X_{2}$ is an arc with two shrinking endpoints, and $E^{3}-X_{2}$ is not 1-ALG at either endpoint of $X_{2}$; and (3) $X_{3}$ is Gillman's wild simple closed curve [7].

A subset $X$ of $E^{3}$ is a pierced set if it lies on a 2-sphere $S$ in $E^{3}$ which can be pierced by a tame arc at each point of $X$. A subset $X$ of $E^{3}$ is called universally pierced if each 2-sphere in $E^{3}$ which contains $X$ can be pierced by a tame arc at each point of $X$. A point $p$ in a set $X$ is called a shrinking point of $X$ if for every open set $U$ in $E^{3}$ which contains $X-\{p\}$, there is an open set $V$ which contains $X-\{p\}$ such that each loop in $V-X$ can be shrunk to a point in $U-X$.

Cannon [4, Theorem 1.1] has characterized nondegenerate, tame, closed subsets $X$ of spheres in $E^{3}$ with the property that each 2 -sphere which contains $X$ and is locally tame modulo $X$ is locally tame at the points of $X$, too. Such a set is called a taming set and is necessarily universally pierced. Loveland [9, Theorem 1] has shown that if an arc $X$ in $E^{3}$ has a shrinking point $p$ and if $E^{3}-X$ has 1-ALG at $p$, then $X$ is universally pierced. (To say $E^{3}-X$ has 1-ALG at $p$ means for each open set $U$ containing $p$, there is an open set $V$ in $U$ which contains $p$ such that each loop in $V-X$ which bounds homologically in $U-X$ bounds a singular disk in $U-X$.) We show that each arc with two shrinking points is universally pierced. It follows from this result that examples (1) and (2) are universally pierced. We also show there are uncountably many nonequivalently embedded pierced sets in $E^{3}$ that are universally pierced arcs.

2. Preservation of tame arcs and wild arcs. In [3, Theorem 1], Brown shows that if $X$ is a cellular subset of $E^{3}$, then there is a continuous function $f$ of $E^{3}$ onto $E^{3}$ such that $f(X)$ is a point and $f$ is one-to-one on $E^{3}-X$. First we need some lemmas about cellular arcs.

Received by the editors June 24, 1974 and, in revised form, May 22, 1975.

AMS (MOS) subject classifications (1970). Primary 57A10, 55A30.

Key words and phrases. Piercing points, pierced sets, universally pierced arcs, wild arcs, surfaces in $E^{3}$, shrinking points, cellular arcs, Alford's arc.

๑ American Mathematical Society 1976 
LEMMA 1. Suppose $X$ is a cellular arc in $E^{3}$ which has a shrinking point $p$, and let $f$ be a continuous function of $E^{3}$ onto $E^{3}$ such that $f(X)$ is a point and $f$ is one-to-one on $E^{3}-X$. If $B$ is a tame arc in $E^{3}$ such that $p$ is an endpoint of $B$ and $\{p\}=B \cap X$, then $f(B)$ is a tame arc.

Proof. Since $B$ lies on the boundary of a disk, so does $f(B)$. Therefore according to [8, Theorem VI], to show $f(B)$ is tame, we only need to find an arbitrarily small 2 -sphere which has $f(X)$ in its interior and meets $f(B)$ at just one point.

Let $\varepsilon$ be a positive number, and let $G$ be an $\varepsilon$-neighborhood of the point $f(X)$. Since $f$ is continuous, there is an open 3-cell $Z$ containing $X$ such that $f(Z) \subset G$ and $B$ meets the 2 -sphere $\operatorname{Bd}(Z)$. Let $W$ be a tame 3-cell in $Z$ whose interior contains $p$ and whose boundary meets $B$ in exactly one point $b$. We may suppose $\operatorname{Bd}(W)$ is locally polyhedral modulo $b$ [2, Theorem 7].

Let $D$ be a disk in $\operatorname{Bd}(W)-X$ with $b$ in its interior. Let $U=Z-(B \cup$ $D$ ). Since $p$ is a shrinking point of $X$, there is an open set $V$ which contains $X-\{p\}$ such that each loop in $V-X$ can be shrunk to a point in $U-X . X$ is contained in the interior of a polyhedral 3-cell $C$ in $V \cup \operatorname{Int}(W)$ such that $\operatorname{Bd}(C)$ and $\operatorname{Bd}(W)$ are in general position. Therefore $\operatorname{Bd}(C) \cap \operatorname{Bd}(W)$ is the union of finitely many disjoint simple closed curves. Let $F$ be the closure of the component of $\operatorname{Bd}(W)-[\operatorname{Int}(D) \cup \operatorname{Bd}(C)]$ which contains $\operatorname{Bd}(D)$. Each component of $\operatorname{Bd}(F)-\operatorname{Bd}(D)$ is a loop in $V-X$ and therefore bounds a singular disk $E_{j}$ in $U-X$. It follows from Dehn's lemma [12] that the singular 2-sphere $D \cup F \cup\left(\cup E_{j}\right)$ can be approximated by a 2 -sphere $S$ which lies in $Z-X$, meets $B$ at just the point $b$, and contains a neighborhood of $b$ in $\operatorname{Bd}(W) . X$ lies in the interior of $S$ because the $\operatorname{arc} B$ meets this 2 -sphere at just the point $b$ and also meets $E^{3}-Z$. It follows that the 2-sphere $f(S)$ has $f(X)$ in its interior and meets $f(B)$ at just the point $f(b)$. Since $S$ is a subset of $Z, f(S)$ is contained in $G$. Therefore $f(B)$ is tame.

REMARK. It follows from [11, Theorem 25] and the proof of Lemma 1 that if an $\operatorname{arc} X$ in $E^{3}$ has a shrinking point, then $X$ is cellular.

LEMMA 2. Suppose $X$ is a cellular arc in $E^{3}$ and let $f$ be a continuous function of $E^{3}$ onto $E^{3}$ such that $f(X)$ is a point and $f$ is one-to-one on $E^{3}-X$. If $C$ is an arc with one wild point $w$ such that $w$ is an endpoint of $C$ and $\{w\}=C \cap$ $X$, then $f(C)$ is a wild arc.

Proof. On the contrary, assume $f(C)$ is tame. Let $S$ be an arbitrary polyhedral 2-sphere whose interior contains $w$. There exist finitely many disjoint polyhedral disks $D_{1}, D_{2}, \ldots, D_{n}$ in $S$ the union of whose interiors contains $S \cap X$ and misses $C$ [11, Theorem 25]. Let

$$
U=\left(E^{3}-S\right) \cup\left(\cup \operatorname{lnt}\left(D_{i}\right)\right) .
$$

Since $f$ is continuous and $f(C)$ is tame, there is a tame 2-sphere $S^{\prime}$ pierced by $f(C)$ at a point $x$ such that $f^{-1}\left(S^{\prime}\right)$ lies in the open set $U, w$ lies in $\operatorname{Int}\left(f^{-1}\left(S^{\prime}\right)\right), f^{-1}(x)$ lies in $\operatorname{Int}(S)$, and, by [2, Theorem 7], $f^{-1}\left(S^{\prime}\right) \cap S$ is the union of finitely many disjoint polyhedral simple closed curves in $U \operatorname{Int}\left(D_{i}\right)$. Let $E$ be the component of $f^{-1}\left(S^{\prime}\right)-S$ which contains $f^{-1}(x)$. Assuming $f^{-1}\left(S^{\prime}\right)$ and $\cup D_{i}$ to be in general position and using the usual "disk 
replacement" technique characteristic of $E^{3}$, we may obtain a 2-sphere $S^{\prime \prime}$ containing a neighborhood of $f^{-1}(x)$ in $E$. The sphere $S^{\prime \prime}$ can be constructed to lie in $\operatorname{Int}(S)$ with $w \in \operatorname{Int}\left(S^{\prime \prime}\right)$ and $S^{\prime \prime} \cap C=\left\{f^{-1}(x)\right\}$. This shows $C$ is tame $[8$, Theorem VI], a contradiction. Therefore $f(C)$ is wild.

LEMMA 3. If $X$ is an arc in $E^{3}$ with a shrinking point $p$ and if $S$ is a 2-sphere containing $X$ that is locally tame modulo $X$, then $S$ can be pierced by a tame arc at each point of $X-\{p\}$.

Proof. Let $f$ be a continuous function of $E^{3}$ onto $E^{3}$ such that $f(X)$ is a point and $f$ is one-to-one on $E^{3}-X$. According to [10, Theorem 2], the conclusion follows if $S$ cannot be pierced by a tame arc at $p$. Therefore we consider the case when $S$ can be pierced by a tame arc at $p$. Let $x$ be a point of $X-\{p\}$, and let $C$ be an arc on $S$ such that $C \cap X$ is an endpoint $x$ of $C$. It follows from [6, Theorem 6] and [5, Theorem 2] that there is a tame arc $B$ in $S$ such that $B \cap X$ is the endpoint $p$ of $B$. By Lemma $1, f(B)$ is a tame arc. But according to [5, Theorem 2], $f(C)$ must be a tame arc because both $f(B)$ and $f(C)$ contain the point $f(X)$ and lie on the 2-sphere $f(S)$. Therefore by Lemma $2, C$ is tame. In this case, $S$ can be pierced by a tame arc at each point of $X$.

3. Arcs with shrinking points. Next we give some examples whose construction involves Alford's wild arc [1].

EXAMPLE (1). Let $X_{1}$ be an arc that is the union of a point $q$ and a null sequence $A_{1}, A_{2}, \ldots$ of copies of Alford's arc converging to $q$ such that each $A_{i} \cap A_{i+1}$ is the shrinking endpoint of $A_{i+1}$ but is the nonshrinking endpoint of $A_{i}$. Eyebolts in the construction of $A_{i}$ should not link those in the construction of $A_{j}(i \neq j) . X_{1}$ has two shrinking endpoints and $E^{3}-X_{1}$ is 1-ALG at $q$.

Example (2). Let $X_{2}$ be an arc that is the union of two copies $A_{1}, A_{2}$ of Alford's arc such that $A_{1} \cap A_{2}$ is the nonshrinking endpoint of $A_{1}$ and $A_{2}$. Eyebolts in the construction of $A_{1}$ should not link those in the construction of $A_{2} . X_{2}$ has two shrinking endpoints and $E^{3}-X_{2}$ is 1-ALG at neither of these endpoints.

EXAMPLE (3). Let $X_{3}$ denote Gillman's simple closed curve [7, p. 452].

According to the next two results, these three examples are universally pierced, although Theorem 1 of [9] can be used to see that $X_{1}$ is universally pierced.

THEOREM 1. Each arc $X$ in $E^{3}$ with two shrinking points is universally pierced.

Proof. Whenever there is a 2-sphere $S$ containing $X$ which is locally tame modulo $X, S$ can be pierced by a tame arc at each point of $X$ according to Lemma 3. Therefore $X$ is universally pierced [9, Lemma 1].

THEOREM 2. Gillman's simple closed curve $X_{3}$ is universally pierced.

Proof. Let $S$ be a 2-sphere containing $X_{3}$ that is locally tame modulo $X_{3}$, and let $x$ be an element of $X_{3}$. There is an arc $A$ in $X_{3}$ such that $A$ contains $x$ in its interior and has a shrinking endpoint. By Lemma 3, $S$ can be pierced by 
a tame arc at $x$. Therefore $X_{3}$ is universally pierced [9, Lemma 1].

Loveland [9, Theorem 4] has shown the existence of countably infinitely many nonequivalent universally pierced arcs in $E^{3}$. We extend his result by using the fact that Alford's arc pierces no disk [7].

THEOREM 3. There exist uncountably many nonequivalently embedded universally pierced arcs in $E^{3}$.

Proof. There are uncountably many increasing sequences of positive integers. For each such sequence $a_{1}, a_{2}, a_{3}, \ldots$, we can construct an arc $A^{\prime}$ that is the union of a point $p$ and a null sequence of a tame arc, $a_{1}$ copies of Alford's arc, a tame arc, $a_{2}$ Alford arcs, a tame arc, $a_{3}$ Alford arcs, . . . such that (1) each arc in the sequence spans a 3-cell which meets only an endpoint of the preceding arc and of the succeeding arc in the sequence, (2) $A^{\prime}$ lies on a 2-sphere $S$, and (3) there is a tame arc $A^{\prime \prime}$ on $S$ such that $A^{\prime} \cap A^{\prime \prime}=\{p\}$ and $A^{\prime} \cup A^{\prime \prime}$ is an arc $A$. Since the union of a collection of universally pierced sets is universally pierced, it follows from [9, Theorem 2] that the arc $A$ is universally pierced. If $B$ is the corresponding arc constructed for a different increasing sequence $b_{1}, b_{2}, b_{3}, \ldots$ of positive integers, then $A$ and $B$ are not equivalently embedded in $E^{3}$ because for different $n$th terms $a_{n}$ and $b_{n}$, some component of the wild set of $A$ is an arc which pierces a disk at just $a_{n}-1$ points while the corresponding component of the wild set of $B$ is an arc which pierces a disk at just $b_{n}-1$ points [7].

\section{REFERENCES}

1. W. R. Alford, Some "nice" wild 2-spheres in $E^{3}$, Topology of 3-Manifolds and Related Topics (Proc. Univ. of Georgia Inst., 1961), Prentice-Hall, Englewood Cliffs, N. J., 1962, pp. 29-33. MR 25 \# 4504.

2. R. H. Bing, Approximating surfaces with polyhedral ones, Ann. of Math. (2) 65 (1957), 456-483. MR 19, 300.

3. Morton Brown, A proof of the generalized Schoenflies theorem, Bull. Amer. Math. Soc. 66 (1960), 74-76. MR 22 \#8470b.

4. J. W. Cannon, Characterization of taming sets on 2-spheres, Trans. Amer. Math. Soc. 147 (1970), 289-299. MR 41 \#2644.

5. P. H. Doyle and J. G. Hocking, Some results on tame disks and spheres in $E^{3}$, Proc. Amer. Math. Soc. 11 (1960), 832-836. MR 23 \# A4133.

6. D. S. Gillman, Side approximation, missing an arc, Amer. J. Math. 85 (1963), 459-476. MR 28 \#3407.

7. , Sequentially 1-ULC tori, Trans. Amer. Math. Soc. 111 (1964), 449-456. MR 28 \#5433.

8. O. G. Harrold, Jr., H. C. Griffith and E. E. Posey, A characterization of tame curves in three-space, Trans. Amer. Math. Soc. 79 (1955), 12-34. MR 19, 972.

9. L. D. Loveland, Some universally pierced arcs in $E^{3}$, Proc. Amer. Math. Soc. 49 (1975), 469-474.

10. D. R. McMillan, Jr., Piercing a disk along a cellular set, Proc. Amer. Math. Soc. 19 (1968), 153-157. MR 36 \#3332.

11. R. L. Moore, Concerning upper semi-continuous collections of continua, Trans. Amer. Math. Soc. 27 (1925), 416-428.

12. C. D. Papakyriakopoulos, On Dehn's lemma and the asphericity of knots, Ann. of Math. (2) 66 (1957), 1-26. MR 19, 761.

13. Harvey Rosen, Piercing points on a special arc, Proc. Amer. Math. Soc. 46 (1974), 438-442. MR 50 \#8498. 